\title{
RELACIÓN DEL CLIMA ORGANIZACIONAL CON LA SATISFACCIÓN LABORAL EN \\ UNA EMPRESA DEL SECTOR DE LA CONSTRUCCIÓN.
}

\section{RELATIONSHIP OF THE ORGANIZATIONAL CLIMATE WITH THE JOB SATISFACTION IN A COMPANY OF THE CONSTRUCTION SECTOR.}

\begin{abstract}
José Gregorio Noboa Salazar, Mgs.
Magíster en Dirección del Talento Humano (Ecuador). Docente a tiempo completo de la Universidad Tecnológica ECOTEC, Ecuador. inoboa@ecotec.edu.ec

Geovanny Javier Barrera Cosiun, Mgs. Magíster en Dirección del Talento Humano (Ecuador). Docente de la Universidad de Espíritu Santo, Ecuador. gbarrera@uees.edu.ec
\end{abstract}

Diana Rojas Torres, Ph.D. Doctor in Management, Innovation, Services and Sustainability (Italia). Docente de la Universidad de La Sabana, Colombia. diana.rojas7@unisabana.edu.co

\section{ARTÍCULO DE INVESTIGACIÓN}

Recibido: 10 de septiembre de 2018.

Aceptado: 19 de noviembre de 2018.

\section{RESUMEN}

El propósito de esta investigación es establecer cómo se vincula el clima organizacional y la satisfacción laboral en una empresa ecuatoriana del sector de la construcción. Como herramienta para determinar los factores del clima organizacional se utilizó el instrumento previamente validado por Ortega-Santos (2018) y en el caso de la medición de los factores de satisfacción laboral se implementó el instrumento Minnesota Satisfaction Questionari (MSQ). Los dos instrumentos se aplicaron a 250 trabajadores en una empresa del sector de la construcción en Ecuador. Metodológicamente, se llevó a cabo un estudio correlacional entre las dimensiones de clima y satisfacción. Se encontró que hay una relación positiva entre 
ambos constructos, concluyendo que las dimensiones del clima organizacional que tienen mayor incidencia sobre la satisfacción laboral son: recompensa y liderazgo. Estudios de este orden son relevantes para las organizaciones ya que les permite proponer y ejecutar planes de acción para mejorar el clima organizacional y la satisfacción laboral en sus empleados.

\section{ABSTRACT}

The purpose of this research is to establish how the organizational climate and job satisfaction in an Ecuadorian company in the construction sector are linked. As a tool to determine organizational climate factors, the instrument previously validated by Ortega-Santos (2018) was used and in the case of the measurement of job satisfaction factors, the Minnesota Satisfaction Questionari (MSQ) instrument was implemented. The two instruments were applied to 250 workers in a company in the construction sector in Ecuador. Methodologically, a correlation study was carried out between the dimensions of climate and satisfaction. It was found that there is a positive relationship between both constructs, concluding that the dimensions of the organizational climate that have the greatest impact on job satisfaction are: reward and leadership. Studies of this order are relevant for organizations since it allows them to propose and execute action plans to improve the organizational climate and job satisfaction in their employees.

Palabras clave: clima organizacional; satisfacción laboral; sector de construcción; análisis correlacional.

\section{INTRODUCCIÓN}

El recurso humano se constituye como una ventaja competitiva en las organizaciones y permite diferenciarlas dentro de una sociedad, siendo éste el intangible más importante para la organización (Llanos, 2016). La personalidad de una organización que influye en el comportamiento del trabajador es conocido como clima organizacional, este establece los procesos organizativos de gestión, cambio e innovación, la calidad de la atención y de los productos y/o servicios prestados, así como en la satisfacción laboral (Elgegren, 2015).

El clima organizacional se centra en las percepciones que una persona tiene respecto a su trabajo, sin embargo, lo que produce satisfacción o insatisfacción en el trabajo dependen de las expectativas que se cree que el trabajo pueda proporcionar (Lavarello \& Gonzales, 2015).

Siendo diferentes el clima y la satisfacción laboral, están íntimamente relacionados; el primero hace referencia a información relacionada a atributos de la empresa, mientras que la 
satisfacción laboral se enfoca en la actitudes y percepciones que tienen los individuos hacia su trabajo (Ramos \& Tejera, 2017).

Analizar el clima organizacional y la satisfacción laboral es relevante para conocer el comportamiento del personal que labora en una empresa, ya que se ha demostrado que son factores determinantes en la eficacia administrativa, pues el comportamiento del grupo está condicionado con la percepción que tiene éste de la organización, por lo que un buen clima organizacional debe ser desarrollado para lograr un equilibrio en las relaciones interpersonales de los colaboradores (Veloso, Cuadra, Gil, Quiroz, \& Meza, 2015).

El sector de la construcción tiene unas características laborales particulares como por ejemplo; mayor accidentalidad laboral debido al tipo de trabajo que realizan los constructores, alta rotación del personal que labora en la construcción, por ser trabajos a corto tiempo o contratos por servicios mientras se ejecuta una obra, alto grado de ausentismo, por la falta de compromiso con el trabajo ya que si al trabajador le ofrecen una nueva oportunidad laboral donde le remuneren mejor cambia sin avisar a su supervisor.

Factores como los anteriormente mencionados, así como la falta de acceso a los servicios de seguridad social, falta de protección de riesgos laborales, la puntualidad en el pago de salarios, el trato humano y el ambiente de trabajo; generan descontento provocando inestabilidad laboral. Por lo tanto, dicha particularidad en el sector de construcción, nos lleva como investigadores a interesarnos en estudiar el clima organizacional y la satisfacción laboral en una empresa en Ecuador con el fin de encontrar las dimensiones del clima organizacional en las cuales se debe trabajar para hacer una transformación en el sector y cómo de manera estratégica los gerentes de recursos humanos deben priorizar a los trabajadores en sus organizaciones ya que como lo afirma (Pérez, Carranza, \& Frías, 2015), la contratación de personal eventual genera más costos administrativos.

El propósito de esta investigación es establecer cómo se vincula el clima organizacional y la satisfacción laboral en una empresa ecuatoriana del sector de la construcción. Para ello, lo primero es investigar mediante revisión teórica, los conceptos de clima organizacional y satisfacción laboral. Luego conocer las particularidades de este sector, y señalar los factores que influyen en el clima y la satisfacción laboral con la finalidad de considerar los resultados hallados luego de la utilización de las herramientas que ayudan a conseguir el objetivo de esta investigación.

La empresa tiene 23 años en el mercado, comercializa equipos, repuestos y servicio técnico para la construcción. Brinda un servicio ágil y oportuno, mejorando continuamente para la 
satisfacción de los clientes. En más detalle, a lo largo de esta investigación, se discutirán las dimensiones que integran al clima organizacional y la satisfacción laboral en el sector de la construcción; relacionando los factores que influyen en las variables estudiadas, permitiendo reconocer situaciones de mejora/cambio en las organizaciones. Finalmente se realizarán unas recomendaciones para los gerentes que laboren en el sector de la construcción teniendo en cuenta los resultados encontrados.

\section{REVISIÓN LITERARIA}

\subsection{Clima Organizacional}

Según Lavarello \& González (2015), el clima tiene que ver con los factores ambientales experimentados conscientemente por los individuos dentro de sus sitios de trabajo. (Zenteno \& Durán, 2016), explica a esta variable como un conjunto de propiedades del ambiente laboral que el trabajador percibe acerca de las estructuras y procesos organizacionales (Graciano, Muñoz, \& Torres, 2014) y de la convergencia entre características personales y organizacionales (Bravo \& Calderón, 2017) que afectan, directa o indirectamente, el comportamiento de las personas dentro de una organización. A su vez, el comportamiento organizacional es la respuesta directa al comportamiento administrativo y las condiciones organizacionales (Cardona \& Zambrano, 2014). Con un resultado positivo o negativo, el clima organizacional influye en los procesos organizacionales y psicológicos.

\subsection{Factores que influyen en el Clima Organizacional.}

Existen varios factores que pueden influir en el clima organizacional de las empresas. Entre ellos podemos mencionar: la cultura organizacional, la motivación, la calidad de la vida laboral y el liderazgo. El clima laboral se relaciona con el desempeño óptimo de los colaboradores de una empresa, puntualizando "conciliación del trabajo con la vida familiar, prestaciones de tipo social, satisfacción en el puesto de trabajo, calidad directiva (liderazgo)" (Zenteno \& Durán, 2016).

\subsection{Satisfacción Laboral}

La Satisfacción en el trabajo se ha considerado importante en la economía (Gomez \& Borra, 2012), en la sociología (Dobrow Riza, Ganzach, \& Liu, 2015) y en la psicología (Veloso, Cuadra, Gil, Quiroz, \& Meza, 2015). Desde los años setenta, la literatura sobre la satisfacción laboral ha aumentado considerablemente. Se pueden distinguir tres corrientes principales de la investigación sobre la satisfacción laboral. La que tiene que ver con las diferencias de género (Zou, 2015), la relación entre la satisfacción laboral (Sánchez-Sellero \& SánchezSellero, 2016), los salarios y la educación (López, 2018). 
La tabla 1 presenta las definiciones de Satisfacción laboral.

Tabla 1. Satisfacción Laboral: conceptos.

\begin{tabular}{|l|c|}
\hline \multicolumn{1}{|c|}{ DEFINICIÓN } & AUTOR \\
\hline $\begin{array}{l}\text { "Estado emocional positivo o placentero } \\
\text { resultante de una percepción subjetiva } \\
\text { de las experiencias laborales del sujeto". }\end{array}$ & (Locke, 1976) \\
\hline $\begin{array}{l}\text { Predisposición a responder de forma } \\
\text { favorable o desfavorable a personas u } \\
\text { objetos del entorno }\end{array}$ & (Navarro, 2010) \\
\hline $\begin{array}{l}\text { Estado emocional, sentimientos o } \\
\text { respuestas afectivas }\end{array}$ & (Chiang \& Ojeda, 2011) \\
\hline Es la actitud ante el trabajo. & (Veloso, Cuadra, Gil, Quiroz, \& Meza, 2015) \\
\hline
\end{tabular}

Fuente: Elaboración propia.

\subsection{Relación entre clima organizacional y satisfacción laboral.}

De acuerdo con Peña, Díaz \& Carrillo (2015), mucho se ha dedicado al estudio sobre el clima organizacional y la satisfacción laboral, tanto de manera individual, como en sus relaciones, y sus efectos sobre la evolución de las empresas. Cuando el empleado ve que sus expectativas no se cumplen en el entorno laboral (clima), surge la insatisfacción laboral; ésta, conduce a la disminución de la productividad de la fuerza laboral, el compromiso organizacional y el compromiso con el trabajo (Méndez, 2015). Además, las condiciones médicas de los empleados pueden verse afectadas negativamente. Se ha observado que una menor satisfacción laboral en los servidores provoca neurosis (insomnio y dolor de cabeza) y negatividad emocional (estrés, decepción) (Murillo, Ramírez, Maldonado, \& García, 2014).

Estudios sobre el comportamiento humano han demostrado que cuando un empleado se siente satisfecho y motivado alcanza un desempeño superior en la realización de su trabajo, además de que se ha expuesto consistentemente las relaciones entre el clima unitario $u$ organizacional y los resultados individuales como el rendimiento, la satisfacción, el compromiso, la implicación y los accidentes (Manosalvas, Manosalvas, \& Quintero, 2015).

(Pérez, Carranza, \& Frías, 2015) centro su estudio en medir la percepción del clima organizacional y la satisfacción laboral en una empresa electrónica, encontrando que la 
dimensión de clima que tenía el mayor impacto en la satisfacción laboral era las relaciones interpersonales.

Los gerentes, buscan establecer buenas interrelaciones bajo un clima organizacional que permita motivar al personal bajo su mando y así mejorar su desempeño, interés y satisfacción en el trabajo, de esta manera el personal tiende a ser más cooperador, adaptable y dispuesto al cambio.

\subsection{Instrumentos para medir clima laboral.}

Existe una gran variedad de instrumentos que miden el clima organizacional, la clave entonces está en encontrar el instrumento que cumpla con el propósito de medición que se tiene en la organización (Ramos \& Tejera, 2017).

Por lo tanto, medir el clima organizacional es una tarea que le ha llevado tiempo a los investigadores ya que las particularidades de cada sector pueden modificar las dimensiones del clima organizacional y por ello, distintos autores se han dedicado a proponer instrumentos de acuerdo al sector donde se requiere llevar a cabo la investigación. Por ejemplo, en el sector de servicios (Araújo, La Torre, López, \& Bastos, 2011) en su estudio analiza factores tales como el estilo de gerencia, retribuciones, la independencia en el trabajo, el salario y los métodos de progreso, estos factores fueron tomados de una recopilación de varios autores (Moss \& Insel, 1974) (Koys \& Decottis, 1991).

Otro instrumento utilizado para medir clima es el de (Ortega, Duque, \& Grueso, 2016), el cual adapta seis dimensiones del estudio de (Weisbord, 1976), para analizar el clima laboral estos son: propósitos u objetivos (que se define según el tipo de organización), relaciones interpersonales (el rol que se desempeña en los problemas entre trabajadores), la distribución del trabajo, componentes de ayuda (tecnológicos, procesos, procedimientos), las recompensas y el liderazgo (los métodos utilizados para la dirección de la organización).

Finalmente, (Añaños, Gutiérrez, \& Rengifo, 2017) toma en cuenta consideraciones como estructura interna de la organización, el apoyo recibido por los trabajadores las recompensas por el trabajo y los riesgos que se corren en este ámbito laboral, el cual es una adaptación del instrumento de (Litwin \& Stringer, 1968).

\subsection{Instrumentos para medir satisfacción laboral.}

La satisfacción laboral al igual que el clima tiene muchos factores que la definen, estos factores permiten realizar una medición del nivel de satisfacción presente en trabajadores de 
una organización, diversos autores han diseñado instrumentos con la finalidad de medir el grado de satisfacción en diferentes ámbitos de la cultura de las organizaciones.

La investigación realizada por (Juárez-Adauta, 2012), utiliza un método de medición de satisfacción laboral basada en una combinación de técnicas de Likert y Thurstone (Bozal, 2006) en el cual se hace una combinación de escalas de los dos instrumentos y se mide en función de respuestas directas sí o no ó 0-1 a las respuestas obtenidas.

Otro instrumento muy utilizado es el Minnesota Satisfaction Questionnarie MSQ (Weiss, Dawis, England, \& Lofquist, 1967), en el cual se miden experiencias de los trabajadores en 5 escalas que integran factores como: la supervisión, la independencia, el estatus social, condiciones de trabajo, la creatividad y la libertad de ejercer su profesión en su versión completa este cuestionario tiene 100 ítems. En la aplicación más común se encuentra la versión corta de 20 ítems, estos están agrupados en dos escalas que son satisfacción intrínseca y extrínseca.

\subsection{Satisfacción y clima laboral en el sector de la construcción.}

(Ordoñez, 2016) en su estudio enfocado en el sector de la construcción resalta factores como el índice de accidentalidad, el cual al aumentar genera un alto costo para las empresas y para el estado. Este índice de accidentes es determinado por las condiciones físico-ambientales en las cuales se desarrolla el trabajo, en ese estudio se realiza una clasificación de los riesgos de trabajo en dos categorías, los factores físicos (mecánicos) y los (no mecánicos).

Otro de los factores importantes que genera inconformidad y poca satisfacción es la alta rotación del personal que labora en la construcción, por ser trabajos de poco tiempo o contratos de tiempo definido, los trabajadores acceden a estos puestos, pero saben que la duración es corta. Así mismo; el ausentismo, la falta de seguridad social y la inestabilidad laboral son variables que generan descontento e inconformidad en los trabajadores de este sector empresarial (Roman, 2015).

La disminución de accidentes de trabajo y la generación de recompensas, pagos a tiempo y mejora de las condiciones de trabajo entre otros factores determina un cambio en la percepción del trabajador respecto a su clima organizacional, mejorando aspectos como la producción y desempeño (Espinoza, 2016). 


\section{MATERIALES Y MÉTODOS}

La investigación es cuantitativa de tipo correlacional, ya que se analizó la relación entre los instrumentos de clima organizacional y satisfacción laboral para encontrar las dimensiones en las cuales había una dependencia.

Los datos recolectados se analizaron utilizando estadística descriptiva mediante correlación de Pearson. La encuesta se aplicó a los colaboradores a nivel nacional, vía correo electrónico al universo de la organización, es decir, 250 empleados; de los cuales respondieron 232 trabajadores.

En esta investigación se aplicaron dos instrumentos. El cuestionario de clima organizacional y el Cuestionario de Satisfacción Minnesota, versión corta (MSQ). La correlación lineal permite comprender la relación entre dos variables. Cuando se pretende analizar el clima organizacional y la satisfacción laboral, se obtiene la media por dimensión y tendríamos una nube de puntos que nos indicaría el tipo de relación existente entre ambas variables.

En particular, nos interesa cuantificar la intensidad de la relación lineal entre las dos variables.

El parámetro que nos permite tal cuantificación es el coeficiente de correlación lineal de Pearson $r$, cuyo valor oscila entre $-1 \mathrm{y}+1$.

El cuestionario de clima organizacional mide 6 dimensiones: "propósitos u objetivos, relaciones entre miembros, la estructura y mecanismos de soporte de la gestión, mecanismos de apoyo, recompensas y el liderazgo" (Ortega, 2016).

La tabla 2 presenta las propiedades psicométricas del cuestionario utilizado.

Tabla 2. Propiedades psicométricas del cuestionario Ortega 2016.

\begin{tabular}{|l|l|l|l|l|l|}
\hline \multicolumn{1}{|c|}{$\begin{array}{c}\text { Análisis de fiabilidad por } \\
\text { dimensión }\end{array}$} & $\begin{array}{l}\text { Kaiser-Mer- } \\
\text { Olkin. }\end{array}$ & $\begin{array}{c}\text { Chi- } \\
\text { cuadro }\end{array}$ & \multicolumn{1}{|c|}{$\begin{array}{c}\text { Alfa } \\
\text { Cronbac } \\
\mathbf{h}\end{array}$} & $\begin{array}{c}\text { Elemento } \\
\text { s }\end{array}$ \\
\hline Propósitos u objetivos &, 923 & 2400 & 28 & 0,923 & 8 \\
\hline Relaciones entre miembros &, 933 & 4881 & 91 & 0,945 & 14 \\
\hline $\begin{array}{l}\text { La estructura y mecanismos de } \\
\text { soporte de la gestión }\end{array}$ &, 882 & 2032 & 36 & 0,883 & 9 \\
\hline Mecanismos de apoyo &, 896 & 3477,72 & 55 & 0,924 & 11 \\
\hline
\end{tabular}




\begin{tabular}{|l|l|l|l|c|l|}
\hline Recompensas &, 842 & 1383,19 & 15 & 0,879 & 6 \\
\hline El liderazgo &, 914 & 1837,65 & 36 & 0,887 & 9 \\
\hline ESCALA TOTAL & & & $\mathbf{0 , 9 7 6}$ & $\mathbf{5 7}$ \\
\hline
\end{tabular}

Nota. Fuente: Ortega, Duque (2016). Los empleados: análisis para dos instituciones de educación superior [IES] en Ecuador. Tesis doctoral.

El Cuestionario de Satisfacción de Minnesota (MSQ) (Weiss, Dawis, England, \& Lofquist, 1967) está diseñado para medir la satisfacción del empleado con su trabajo. Esta escala se presenta en dos formatos: una versión larga de 100 ítems y otra abreviada de 20; en ambas ediciones se incluyen ítems en los que se interroga acerca de 20 facetas de la satisfacción laboral, aunque las puntuaciones de estas se calculan solo para el formato largo. En el formato corto se evalúa la satisfacción general, la intrínseca (naturaleza del puesto) y la extrínseca (otros aspectos de la situación del trabajo, como las prestaciones y el salario) (anexo 2).

\section{RESULTADOS}

Los datos recolectados se analizaron mediante correlación de Pearson (utilizando el programa estadístico STATA 12), confirmando que 'los datos tienen una distribución normal. Luego de la recolección de los datos, se hizo el tratamiento de los datos utilizando estadística paramétrica.

\subsection{Información Sociodemográfica}

Los cuestionarios fueron respondidos por 232 trabajadores, pertenecientes al área administrativa, financiera, producción, ventas y servicios.

Tabla 3. Estadísticos descriptivos del instrumento de clima organizacional.

\begin{tabular}{|c|c|c|}
\hline Variable & Descripción & $(\%)$ \\
\hline \multirow{2}{*}{ Sexo } & Masculino & $61,6 \%$ \\
\hline & Femenino & $38,4 \%$ \\
\hline \multirow{2}{*}{ Edad } & 18 - 24 años & $21,1 \%$ \\
\hline & 25 - 29 años & $39,7 \%$ \\
\hline
\end{tabular}




\begin{tabular}{|llr|}
\hline & 30 - 39 años & $32,8 \%$ \\
& $40-$ en adelante & $6,5 \%$ \\
\hline \multirow{4}{*}{ Área } & Administración & $16,4 \%$ \\
& Auditoría & $2,2 \%$ \\
& Desarrollo Humano & $3,5 \%$ \\
& Finanzas & $9,9 \%$ \\
& Maquinarias & $13,8 \%$ \\
& Post Venta & $18,8 \%$ \\
& Servicios & $30,6 \%$ \\
Años laborando & Sistema de Gestión & $3,9 \%$ \\
& Menos de 1 año & $19,4 \%$ \\
& Entre 1 -4 años & $45,0 \%$ \\
& Entre 5 -9 años & $28,5 \%$ \\
& Más de 10 años & $5,2 \%$ \\
\hline
\end{tabular}

Fuente: Elaboración propia

La variable sexo; el $62 \%$ son hombres y el $38 \%$ mujeres, lo que significa que en el sector de la construcción se contrata más hombres para cargos operativos como: técnicos, mecánicos, eléctricos, choferes; en los que se da preferencia la contratación de hombres mientras que las mujeres se contratan por lo general, para cargos administrativos.

Se evidencia que sólo el 6,5\% de los colaboradores tiene una edad de 40 años en adelante, lo que evidencia que el sector de construcción contrata colaboradores jóvenes según sus necesidades de esfuerzo físico, debido al giro de negocio.

El 31\% de la estructura de la empresa pertenece al área de servicios, ya que en la empresa su ventaja competitiva radica en la atención, retención y fidelización de los clientes.

Se puede apreciar que sólo el 5,2\% de colaboradores tienen más de 10 años de antigüedad, lo que demuestra que la empresa es muy estable para cargos administrativos e inestables para cargos operativos, por su alta rotación.

\subsection{Clima Organizacional}

El cuestionario de 57 preguntas de EJDO, midió la percepción de clima organizacional de los trabajadores de la empresa respecto a seis dimensiones: Propósitos u objetivos, relaciones entre miembros, estructura y mecanismos de soporte de la gestión, mecanismos de apoyo, 
recompensas y liderazgo. Las variables se midieron en una escala de Likert de 7 puntos; siendo 1 totalmente en desacuerdo y 7 totalmente de acuerdo.

Tabla 4. Estadísticos descriptivos en el clima organizacional.

\begin{tabular}{|c|c|c|c|c|c|}
\hline \multicolumn{6}{|c|}{ Propósitos u objetivos } \\
\hline Variable & Obs. & Media & Std. Desv. & Mín. & Max \\
\hline $\mathrm{P} 1$ & 210 & 5,7 & 1,6 & 1 & 7 \\
\hline $\mathrm{P} 2$ & 210 & 5,9 & 1,4 & 1 & 7 \\
\hline P3 & 209 & 5,6 & 1,5 & 1 & 7 \\
\hline P4 & 208 & 5,7 & 1,5 & 1 & 7 \\
\hline P5 & 209 & 5,7 & 1,5 & 1 & 7 \\
\hline P6 & 209 & 5,8 & 1,4 & 1 & 7 \\
\hline P7 & 209 & 5,6 & 1,6 & 1 & 7 \\
\hline P8 & 208 & 5,5 & 1,9 & 1 & 7 \\
\hline \multicolumn{6}{|c|}{ Relaciones entre miembros de la organización } \\
\hline \multicolumn{6}{|l|}{ Comunicación } \\
\hline Variable & Obs. & Media & Std. Desv. & Mín. & Max. \\
\hline P9 & 210 & 6,0 & 1,4 & 1 & 7 \\
\hline P10 & 210 & 6,2 & 1,1 & 1 & 7 \\
\hline P11 & 209 & 6,2 & 1,2 & 1 & 7 \\
\hline P12 & 210 & 5,9 & 1,4 & 1 & 7 \\
\hline P13 & 210 & 5,9 & 1,5 & 1 & 7 \\
\hline \multicolumn{6}{|c|}{ Trato interpersonal } \\
\hline Variable & Obs. & Media & Std. Desv. & Mín. & Max. \\
\hline P14 & 210 & 6,2 & 1,2 & 1 & 7 \\
\hline P15 & 210 & 6,1 & 1,3 & 1 & 7 \\
\hline P16 & 210 & 6,0 & 1,4 & 1 & 7 \\
\hline P17 & 210 & 5,9 & 1,6 & 1 & 7 \\
\hline P18 & 209 & 6,0 & 1,2 & 1 & 7 \\
\hline \multicolumn{6}{|c|}{ Manejo de conflictos } \\
\hline Variable & Obs. & Media & Std. Desv. & Mín. & Max. \\
\hline P19 & 210 & 6,2 & 1,3 & 1 & 7 \\
\hline P20 & 209 & 6,3 & 1,2 & 1 & 7 \\
\hline P21 & 210 & 6,3 & 1,1 & 1 & 7 \\
\hline
\end{tabular}




\begin{tabular}{|c|c|c|c|c|c|}
\hline P22 & 209 & 6,2 & 1,3 & 1 & 7 \\
\hline \multicolumn{6}{|c|}{ La estructura y mecanismos de soporte de la gestión } \\
\hline \multicolumn{6}{|c|}{ Espacios infraestructura y lugar de trabajo } \\
\hline Variable & Obs. & Media & Std. Desv. & Mín. & Max. \\
\hline P23 & 210 & 6,2 & 1,3 & 1 & 7 \\
\hline P24 & 209 & 6,3 & 1,2 & 1 & 7 \\
\hline P25 & 209 & 6,1 & 1,4 & 1 & 7 \\
\hline P26 & 208 & 5,8 & 1,7 & 1 & 7 \\
\hline \multicolumn{6}{|c|}{ Estructura, jerarquía y responsabilidades } \\
\hline Variable & Obs. & Media & Std. Desv. & Mín. & Max. \\
\hline P27 & 209 & 5,9 & 1,3 & 1 & 7 \\
\hline P28 & 210 & 6,1 & 1,3 & 2 & 7 \\
\hline P29 & 210 & 6,0 & 1,4 & 1 & 7 \\
\hline P30 & 210 & 6,1 & 1,3 & 1 & 7 \\
\hline P31 & 209 & 6,0 & 1,4 & 1 & 7 \\
\hline \multicolumn{6}{|c|}{ Mecanismos de apoyo } \\
\hline \multicolumn{6}{|c|}{ Tecnologías de soporte y herramientas tecnológicas } \\
\hline Variable & Obs. & Media & Std. Desv. & Mín. & Max \\
\hline P32 & 209 & 5,9 & 1,6 & 1 & 7 \\
\hline P33 & 209 & 6,1 & 1,4 & 1 & 7 \\
\hline P34 & 207 & 5,9 & 1,6 & 1 & 7 \\
\hline P35 & 209 & 5,9 & 1,6 & 1 & 7 \\
\hline P36 & 209 & 6,0 & 1,4 & 1 & 7 \\
\hline P37 & 209 & 6,0 & 1,5 & 1 & 7 \\
\hline \multicolumn{6}{|c|}{ Procesos, procedimientos y funciones } \\
\hline Variable & Obs. & Media & Std. Desv. & Mín. & Max. \\
\hline P38 & 209 & 6,0 & 1,4 & 1 & 7 \\
\hline P39 & 209 & 5,8 & 1,6 & 1 & 7 \\
\hline P40 & 209 & 5,8 & 1,5 & 1 & 7 \\
\hline P41 & 209 & 5,7 & 1,7 & 1 & 7 \\
\hline P42 & 208 & 5,5 & 1,8 & 1 & 7 \\
\hline \multicolumn{6}{|c|}{ Recompensas } \\
\hline Variable & Obs. & Media & Std. Desv. & Mín. & Max. \\
\hline P43 & 210 & 5,7 & 1,8 & 1 & 7 \\
\hline P44 & 207 & 5,6 & 1,8 & 1 & 7 \\
\hline
\end{tabular}




\begin{tabular}{|c|ccc|c|c|}
\hline P45 & 210 & 5,3 & 2,1 & 1 & 7 \\
P46 & 210 & 5,6 & 1,8 & 1 & 7 \\
P47 & 210 & 5,5 & 2,0 & 1 & 7 \\
P48 & 209 & 5,9 & 1,6 & 1 & 7 \\
\hline Liderazgo & Obs. & Media & Std. Desv. & Mín. & Max. \\
\hline Variable & 210 & 5,8 & 1,6 & 1 & 7 \\
\hline P49 & 209 & 5,9 & 1,5 & 1 & 7 \\
P50 & 210 & 5,5 & 1,8 & 1 & 7 \\
P51 & 209 & 5,8 & 1,4 & 1 & 7 \\
P52 & 209 & 6,5 & 1,1 & 1 & 7 \\
P53 & 210 & 5,9 & 1,5 & 1 & 7 \\
P54 & 210 & 5,9 & 1,5 & 1 & 7 \\
P55 & 210 & 6,1 & 1,3 & 1 & 7 \\
P56 & 210 & 6,3 & 1,1 & 1 & 7 \\
P57 & & & & & \\
\hline
\end{tabular}

Fuente: Elaboración propia.

Las preguntas que más se destacan por tener calificaciones más altas son: la (P53) y se refiere a que el trabajo en equipo es fundamental para la consecución de resultados, en la dimensión del liderazgo, con una calificación de (6.5).

Adicional se detecta que dentro de la variable manejo de conflictos, las preguntas (P20, P21) que hacen referencia a la organización y a la forma de resolver los problemas a través del dialogo, así como también al compañerismo entre trabajadores, ambas preguntas con una calificación de (6.3).

También se evidencia dentro de las preguntas mejores calificadas, en la variable espacio, infraestructura y lugar de trabajo, se destaca que el trabajador se siente a gusto de trabajar en la empresa, teniendo una calificación de (6.3), que corresponde a la pregunta (P24).

Se detecta también que los colaboradores perciben que su supervisor es competente al ejecutar su trabajo, este enunciado corresponde a la pregunta (P57), con una calificación de (6.3).

Las preguntas con menores calificaciones de parte de los colaboradores fueron la (P45), que se encuentra dentro de la dimensión Recompensas con una calificación de (5.3) y se refiere al percibir que la organización cancela una suma justa por el trabajo que realizan. Así mismo dentro de la dimensión: Propósitos u objetivos, en la pregunta (P8), con una calificación de 
(5.5.), se refiere que la organización siempre comunica a los empleados, los resultados obtenidos en un período anterior.

Se detallan en la Tabla 5, las preguntas que cuentan con mejores percepciones.

Tabla 5. Clima organizacional, factores relevantes.

\begin{tabular}{|c|c|c|c|c|c|}
\hline Variable & Obs. & Media. & Std. Desv. & Mín. & Max. \\
\hline P53 & 209 & 6,5 & 1,1 & 1 & 7 \\
P20 & 209 & 6,3 & 1,2 & 1 & 7 \\
P21 & 210 & 6,3 & 1,1 & 1 & 7 \\
P24 & 209 & 6,3 & 1,2 & 1 & 7 \\
P57 & 210 & 6,2 & 1,1 & 1 & 7 \\
P10 & 210 & 6,2 & 1,1 & 1 & 7 \\
P11 & 209 & 6,2 & 1,2 & 1 & 7 \\
P14 & 210 & 6,2 & 1,2 & 2 & 7 \\
P23 & 210 & 6,2 & 1,3 & 1 & 7 \\
\hline
\end{tabular}

Fuente: Elaboración propia.

En la tabla 6, muestra un resumen de las 6 dimensiones, en la que se destacan como mejores calificadas por los colaboradores, las relaciones entre miembros de la organización y la estructura y mecanismos de soporte de la gestión, ambas con una media de (6.1).

Tabla 6. Estadísticos descriptivos de clima organizacional.

\begin{tabular}{|l|r|r|r|}
\hline \multicolumn{1}{|c|}{ Dimensión } & Obs. & \multicolumn{1}{c|}{ Media } & \multicolumn{1}{c|}{ Std. } \\
\hline PROPÓSITOS U OBJETIVOS & 205 & 5,7 & 1,41 \\
\hline $\begin{array}{l}\text { RELACIONES ENTRE MIEMBROS EN LA } \\
\text { ORGANIZACIÓN }\end{array}$ & 207 & 6,1 & 1,08 \\
\hline $\begin{array}{l}\text { LA ESTRUCTURA Y MECANISMOS DE SOPORTE DE LA } \\
\text { GESTION }\end{array}$ & 204 & 6,1 & 1,12 \\
\hline MECANISMOS DE APOYO & 206 & 5,9 & 1,30 \\
\hline RECOMPENSAS & 206 & 5,6 & 1,63 \\
\hline EL LIDERAZGO & 207 & 6,0 & 1,18 \\
\hline
\end{tabular}

Fuente: Elaboración propia. 


\subsection{Satisfacción laboral}

La satisfacción laboral se determinó mediante el cuestionario corto de Minnesota el cual consta de 20 preguntas, con una escala del 1 al 7 para conocer el nivel de satisfacción de los colaboradores y dos factores; intrínseco y extrínseco.

Tabla 7. Estadísticos descriptivo satisfacción laboral

\begin{tabular}{|l|l|l|l|l|l|}
\hline Variable & Obs. & Media & $\begin{array}{c}\text { Std. } \\
\text { Desv. }\end{array}$ & Mín. & Max. \\
\hline P1 & 203 & 6,59 & 0,71 & 1 & 7 \\
P2 & 203 & 6,48 & 0,96 & 1 & 7 \\
P3 & 202 & 6,25 & 1,23 & 1 & 7 \\
P4 & 202 & 6,22 & 1,32 & 1 & 7 \\
P5 & 203 & 6,13 & 1,25 & 1 & 7 \\
P6 & 203 & 6,09 & 0,96 & 2 & 7 \\
P7 & 201 & 6,33 & 1,08 & 1 & 7 \\
P8 & 203 & 6,22 & 1,22 & 1 & 7 \\
P9 & 203 & 6,21 & 1,36 & 1 & 7 \\
P10 & 203 & 6,26 & 1,12 & 1 & 7 \\
P11 & 203 & 6,43 & 1,00 & 1 & 7 \\
P12 & 202 & 6,17 & 1,17 & 1 & 7 \\
P13 & 203 & 5,41 & 2,03 & 1 & 7 \\
P14 & 201 & 5,78 & 1,72 & 1 & 7 \\
P15 & 203 & 6,10 & 1,37 & 1 & 7 \\
P16 & 203 & 6,15 & 1,36 & 1 & 7 \\
P17 & 203 & 6,17 & 1,23 & 1 & 7 \\
P18 & 203 & 6,42 & 0,85 & 1 & 7 \\
P19 & 203 & 5,91 & 1,61 & 1 & 7 \\
P20 & 202 & 6,31 & 1,22 & 1 & 7 \\
\hline
\end{tabular}

Fuente: Elaboración propia. 
Tabla 8. Estadísticos descriptivo factores motivacionales intrínsecos.

\begin{tabular}{|c|c|c|c|c|c|}
\hline Variable & Obs. & Media & Std. Desv. & Mín. & Max. \\
\hline SANTIN 1 & 203 & 6,59 & 0,71 & 1 & 7 \\
SANTIN 2 & 203 & 6,48 & 0,96 & 1 & 7 \\
SATIN 3 & 202 & 6,25 & 1,23 & 1 & 7 \\
SATIN 4 & 202 & 6,22 & 1,32 & 1 & 7 \\
SATIN 7 & 201 & 6,33 & 1,08 & 1 & 7 \\
SATIN 8 & 203 & 6,22 & 1.22 & 1 & 7 \\
SATIN 9 & 203 & 6,21 & 1,36 & 1 & 7 \\
SATIN 10 & 203 & 6,26 & 1,12 & 1 & 7 \\
SATIN 11 & 203 & 6,43 & 1,00 & 1 & 7 \\
SATIN 15 & 203 & 6,10 & 1,37 & 1 & 7 \\
SATIN 16 & 203 & 6,15 & 1,36 & 1 & 7 \\
SATIN 17 & 203 & 6.17 & 1,23 & 1 & 7 \\
SATIN 18 & 203 & 6.42 & 0.85 & 1 & 7 \\
SATIN 20 & 202 & 6.31 & 1,22 & 1 & 7 \\
\hline
\end{tabular}

Fuente: Elaboración propia.

Tabla 9. Estadísticos descriptivo factores motivacionales extrínsecos.

\begin{tabular}{|c|c|c|c|c|c|}
\hline Variable & Obs & Media & Std. Desv. & Mín & Max \\
\hline SATEX 5 & 203 & 6,13 & 1,25 & 1 & 7 \\
SATEX 6 & 203 & 6.10 & 0.96 & 2 & 7 \\
SATEX 12 & 202 & 6.17 & 1,17 & 1 & 7 \\
SATEX 13 & 203 & 5.41 & 2.03 & 1 & 7 \\
SATEX 14 & 201 & 5.78 & 1,72 & 1 & 7 \\
SATEX 19 & 203 & 5.91 & 1,61 & 1 & 7 \\
\hline
\end{tabular}


Fuente: Elaboración propia.

Tabla 10. Estadísticos descriptivos factores intrínsecos y extrínsecos.

\begin{tabular}{|l|c|c|}
\hline \multicolumn{1}{|c|}{ Stats } & INTRÍNSECOS & EXTRÍNSECOS \\
\hline Mean & 6,31 & 5,91 \\
p50 & 6,57 & 6,5 \\
sd. & 0,85 & 1,20 \\
Varianza & 0,73 & 1,43 \\
N & 198 & 200 \\
Range & 5,57 & 5,0 \\
Min & 1,43 & 2.0 \\
Max & 7,0 & 7,0 \\
\hline
\end{tabular}

Fuente: Elaboración propia.

Tabla 11. Correlación entre clima organizacional y satisfacción laboral.

\begin{tabular}{|l|l|l|l|l|l|l|l|l|}
\hline & PROP & REL & ESTRUC & MEC & REC & LID & INT & EXT \\
\hline PROPOSITO & 1 & & & & & & & \\
\hline RELACIONES & $0,87^{*}$ & 1 & & & & & & \\
\hline ESTRUCTURA & $0,89^{*}$ & $0,89^{*}$ & 1 & & & & & \\
\hline MECANISMOS & $0,91^{*}$ & $0,86^{*}$ & $0,94^{*}$ & 1 & & & & \\
\hline RECOMPENSA & $0,89^{*}$ & $0,83^{*}$ & $0,88^{*}$ & $0,91^{*}$ & 1 & & & \\
\hline LIDERAZGO & $0,89^{*}$ & $0,86^{*}$ & $0,92^{*}$ & $0,93^{*}$ & $0,91^{*}$ & 1 & & \\
\hline INTRINSECO & $0,77^{*}$ & $0,77^{*}$ & $0,84^{*}$ & $0,81^{*}$ & $0,74^{*}$ & $0,82^{*}$ & 1 & \\
\hline EXTRINSECO & $0,88^{*}$ & $0,87^{*}$ & $0,90^{*}$ & $0,90^{*}$ & $0,93^{*}$ & $0,91^{*}$ & $0,82^{*}$ & 1 \\
\hline
\end{tabular}

Nota. Fuente: Elaboración propia. Valores significativos $\mathrm{p}<0.05$ *

La información obtenida en la tabla 11 indica una relación entre factores de satisfacción y clima que tiene mayor fortaleza en el factor intrínseco con las escalas de clima: estructura, mecanismo de apoyo, y liderazgo, con lo que se adecua a lo expuesto por (García , Artacho, Zurita, \& Martos, 2015), los empleados deben sentirse satisfechos con factores relacionados con las relaciones entre miembros, y las relaciones con la gerencia demostrando así que un buen liderazgo influye en la satisfacción si este afecta el clima, en el cual los trabajadores desempeñan sus funciones. 
En el otro lado tenemos la satisfacción extrínseca, esta tiene una correlación significativa y fuerte con estructura y soporte de gestión, mecanismos de apoyo, recompensa y liderazgo, (Volpi, 2014), señala que si un trabajador se siente satisfecho con las recompensas recibidas y su rendimiento es óptimo. Los factores de estructura y soporte y los mecanismos de apoyo son los factores que más influyen en la satisfacción, concuerda con lo expuesto por (Kronberg, 2013), esta autora indica que los factores extrínsecos como la higiene, políticas de la empresa, administración, condiciones laborales dan al trabajador insatisfacción si son manejados de una manera errónea, y crean un ambiente adecuado de satisfacción si el trabajador percibe que estos factores son bien atendidos por la organización.

Cada ítem de motivación se califica considerando una escala de Likert de 1 a 7 , siendo 7 totalmente satisfecho y 1 menos satisfecho, con una media por encima de 5 como se observa en la tabla 10 (Bozal , 2006).

Con una medida superior a 5 en promedio, esto significa que los colaboradores se encuentran satisfechos con el trabajo que están realizando y esto impacta positivamente el clima organizacional.

Relacionando los resultados de la satisfacción laboral con el clima organizacional, encontramos que, de las 57 preguntas del instrumento de clima organizacional, no hay respuestas en el rango de 1-4 en la escala de Likert, en un intervalo de 1-7; lo que ratifica la relación entre éstos dos constructos en el sector de la construcción.

El objetivo de la empresa es mejorar el clima organizacional en busca de la excelencia, por lo que se propone realizar un plan de acción alineado a la estrategia corporativa para fortalecer el clima organizacional y por ende la satisfacción de sus colaboradores.

Por otra parte; los factores extrínsecos son los que tienen menor percepción, aunque tiene una media superior a 5, son los que la compañía deben incluir en el plan de acción para alcanzar la excelencia y estos son: sentirse a gusto con su salario y la cantidad de trabajo que hacen, así como también perciben que tiene limitadas oportunidades de avanzar y consideran que reconocen poco sus esfuerzos, cuando realizan un buen trabajo.

Los factores intrínsecos como la percepción del colaborador de mantenerse ocupado en su trabajo, la oportunidad de poder realizar sus actividades de manera individual sin estar todo el tiempo supervisado por su jefe, demuestran que la compañía confía en las habilidades de sus colaboradores, debido a que tienen colaboradores productivos en cada puesto de trabajo y existe una relación positiva de apertura y confianza entre sus subalternos y superiores. 
Podemos apreciar en la tabla 9 la correlación entre los factores de clima organizacional y los de satisfacción laboral. Se identificó que los factores intrínsecos y extrínsecos, se relacionan de manera significativa con todas las dimensiones del clima organizacional: Propósitos $u$ objetivos, relaciones entre los miembros, estructura y mecanismos de soporte de la gestión, mecanismos de apoyo, recompensas y liderazgo, debido a que su correlación tiene valores mayores a 0.70 .

\section{CONCLUSIONES}

En el presente estudio se ha cumplido con el objetivo general de determinar la relación entre el clima organizacional y la satisfacción laboral en una empresa del sector de la construcción, encontrándose relación directa y significativa entre estos dos constructos.

Para profundizar nuestros conocimientos referentes al clima organizacional y satisfacción laboral, fueron investigados ambos. Identificamos que el clima se centra en la empresa y la gestión de la misma para crear su ambiente laboral, mientras que satisfacción laboral, es la imagen que tiene el colaborador sobre la empresa según su experiencia en el trabajo. La entidad encargada de generar un buen clima hacia sus trabajadores es la empresa, generando satisfacción laboral a sus trabajadores.

Adicional se realizó una investigación mediante revisión teórica, dentro del sector de la construcción de los factores relevantes que impactan tanto en el clima como en la satisfacción laboral y se identificó que existen varias particularidades que afectan la satisfacción laboral, tales como: accidentes laborales, inestabilidad laboral, impuntualidad en el pago de salarios, trato inadecuado que reciben los colaboradores, entre otras.

Se evaluó los resultados de la aplicación de los instrumentos aplicados para evidenciar que la satisfacción laboral tiene un efecto con tendencia positiva sobre el clima organizacional, con oportunidades de mejora que generen estándares de excelencia, motivo por lo cual, se recomienda desarrollar planes de acción que impulsen el mejoramiento del clima y la satisfacción laboral.

Al finalizar la investigación se concluye que los resultados del estudio de clima y satisfacción laboral son buenos, pero existe la posibilidad de realizar mejoras, mediante la elaboración de planes de acción que permitan fortalecer la comunicación a través del desarrollo de campañas de información descendentes sobre los resultados alcanzados en el periodo anterior, así como también, se podría informar a los colaboradores sobre el actual plan de negocio de la empresa. 
Adicional se recomienda realizar un comparativo de sueldos actuales de la compañía con relación al mercado, considerando el sector de la construcción, para confirmar que los ingresos actuales de los colabores son justos por el trabajo realizado.

Con este trabajo se impulsa nuevas líneas de investigación que analicen la relación entre el clima y la satisfacción laboral en otras empresas del sector de la construcción tales como: constructoras, promotoras inmobiliarias, alquiler de maquinarias, servicios de excavación y movimientos de tierra, rehabilitación de edificios, plantas de fabricación de materiales de construcción, servicios arquitectónicos entre otras, adicional se recomienda realizar estudios sobre la justicia organizacional y su relación con el clima organizacional y satisfacción laboral.

\section{REFERENCIAS BIBLIOGRÁFICAS}

Añaños Zegarra, A., Gutierrez Morales, C., \& Rengifo Paredes, M. (2017). CLIMA ORGANIZACIONAL Y SATISFACCIÓN LABORAL EN PEQUEÑAS EMPRESAS DEL SECTOR CONSTRUCCIÓN. CASO PERUANO. Lima: Tesis presentada grado de Magíster en Administración.

Araújo Silva Freire, R. P., La Torre Martínez, J. M., López Hernández, A. M., \& Bastos Paiva, S. (2011). El clima organizacional en el diseño del Balanced Scorecard: Evaluación psicométrica de un instrumento de medida. Contabilidade Vista \& Revista, 22.

Bozal , M. (2006). Escala mixta Likert-Thuretone. ANDULI, Revista Andaluza de Ciencias Sociales, 81-95.

Bravo, M., \& Calderón, J. (2017). El Clima Laboral y su Incidencia en el Desempeño del Personal Docente de una Escuela de Educación Básica en Ecuador. PODIUM Edición

Cardona, D., \& Zambrano, R. (2014). Revisión de instrumentos de evaluación de clima organizacional. Elsevier, 185-187. Obtenido de https://search.proquest.com/docview/1562070794 ?accountid=130858

Chiang, M., \& Ojeda, J. (2011). Estudio de la relación entre satisfacción laboral y productividad de los trabajadores de las ferias libres. Revista Contaduría y Administración, Facultad de Contaduría y Administración. Universidad Nacional Autónoma de México. . 
Dobrow Riza, S., Ganzach, Y., \& Liu, Y. (2015). Time and job satisfaction: a longitudinal study of the differential roles of age and tenure. Journal of Management.

Eagly, A. H., \& Johannesen-Schmidt, M. C. (2001). The leadership styles of women and men. Journal of Social Issues.

Elgegren, U. (julio de 2015). Fortalecimiento del clima organizacional en establecimientos de salud. Tematica psicologica.

Espinoza, C. (2016). Incidencia de la seguridad ociupacional en el sector de la contrucción. Repositorio UESS, 8-16.

García , C., Artacho, C., Zurita, J., \& Martos, F. (2015). Diferencias en afrontamiento, bienestar y satisfacción entre desempleados y empleados en cuanto al género. . Revista electrónica de investigación y docencia creativa.

Gomez, F., \& Borra, C. (2012). SATISFACCIÓN LABORAL Y SALARIO: ¿COMPENSA LA RENTA LABORAL LAS CONDICIONES NO MONETARIAS DEL TRABAJO? Revista de Economía

Graciano, B., Muñoz, L., \& Torres, Y. (2014). DIAGNOSTICO Y EVALUACION DEL CLIMA LABORAL DE LA INSTITUCIÓN EDUCATIVA DANIEL ALFONSO PAZ ÁLVAREZ DEL MUNICIPIO DE LA APARTADA - CÓRDOBA. REPOSITODIO UNIVERSIDAD SAN BUENAVENTURA FUNDACION UNIVERSITARIA CATOLICA DEL NORTE. Obtenido de http://bibliotecadigital.usb.edu.co/bitstream/10819/2276/1/Diagnostico_Clima_Laboral _Graciano_2014.pdf

Juárez-Adauta, S. (2012). Clima organizacional y satisfacción laboral. Revista Médica del Instituto Mexicano del Seguro Social.

Koys, D. J., \& Decottis, T. A. (1991). Inductive measures of psychological climate. Human Relations, 1-3.

Kronberg, I. (2013). Employee Satisfaction on Cruise Ships . Alemania: GRIN Verlag.

Lavarello, J., \& Gonzales, J. (2015). ¿Qué factores de la cultura organizacional se asocian con el bienestar laboral?: Un estudio en una empresa de servicios. Escritos sobre

Litwin, G., \& Stringer, R. (1968). Motivación y clima organizacional. Motivación y clima organizacional. 
Llanos, M. (2016). El desarrollo de los sistemas de producción y su influencia en las relaciones laborales y el rol del trabajador. Economía y Desarrollo, 3-17.

Locke, E. (1976). The nature and causes of job satisfaction. En D. E. Dunnette, Handbook of Industrial and Organizational Psychology. Chicago: Rand McNally.

López, M. P. (2018). Escala para valorar la percepción y grado de satisfacción del profesorado de Educación Primaria de la Comunidad Autónoma de Andalucía respecto a los procesos tutoriales. Complutense de Educación, 288-305. Obtenido de https://search.proquest.com/docview/2050568434 ?accountid=130858

Manosalvas, C., Manosalvas, L., \& Quintero, J. (2015). El clima organizacional y la satisfacción laboral: un análisis cuantitativo riguroso de su relación. AD-minister, 513. Obtenido de https://search.proquest.com/docview/1706514369?accountid=130858

Méndez, M. (2015). Satisfacción con el empleo de extensionista rural: un estudio cualitativo en Caldas, Colombia. Ceres, 242-248. Obtenido de https://search.proquest.com/docview/1718353686 ?accountid=130858

Moss, R., \& Insel, P. (1974). The work Enviroment Scale. Palo Alto, C.A:. En Versión española:

Murillo, M., Ramírez, C., Maldonado, S., \& García, B. (2014). Validación de un instrumento de la calidad de vida laboral en una institución de educación pública a nivel superior en México. International Journal of Arts \& Sciences, 62-77. Obtenido de https://search.proquest.com/docview/1652449677?accountid=130858

Navarro, E. (2010). Satisfacción laboral evocada por los profesionales de la construcción en la comunidad Valenciana. Revista de la Construcción. Vol. 9, 6.7.

Ordoñez, M. (2016). DISEÑO DE MODELO CUANTITATIVO DE RIESGOS LABORALES PARA EL SECTOR DE LA CONSTRUCCIÓN EN EL ECUADOR. Repositorio Universidad de Guayaquil, 8-59.

Ortega Santos, J. P. Incidencia del clima organizacional en la satisfacción laboral de los empleados:

Análisis para dos instituciones de educación superior [IES] en Ecuador (Doctoral dissertation,

Universidad del Rosario). 
Ortega Santos, J. P., Duque Oliva, E. J., \& Grueso Hinestroza, M. P. (2016). Clima Organizacional: Origen, Elucion, Medicion y Aplicaciones en Instituciones Educativas. Uees, Samborondon.

Peña, M., Diaz, M., \& Carrillo , A. (2015). relacion del clima organizacional y satisfaccion laboral en una pequeña empresa familiar. Revista internacional administracion y finanzas, 8(1), 14-37.

Perez, A., Carranza, G., \& Frias, M. (2015). MODELO DE FACTORES EXPLICATIVOS DE CLIMA ORGANIZACIONAL EN LOS MEDIOS DE COMUNICACIÓN DEL ESTADO DE TABASCO. $X X$ Congreso Internacional de Contaduria, Administracion e Informatica, At Ciudad de Mexico, Distrito Federal, Mexico. Mexico: Universitarias.

Ramos, V., \& Tejera, E. (2017). Estudio de relaciones entre cultura, clima y fuerza de clima laboral en Ecuador. Acción Psicológica, 225-240. doi:10.5944

Roman, E. (2015). Estudio de la problematica laboral por la que atraviesan los profesionales dedicados a la contruccion de obras civiles miunicipales de menor cuantia en la ciaudad de Cuenca-Ecuador, al momento de contratar mano de obra y sus prinsipales desafios y amenaza. Repositorio Universidad del Azuay, 38-86.

Sánchez-Sellero, M., \& Sánchez-Sellero, P. (2016). Factores determinantes de la satisfacción laboral en España antes y durante la crisis de 2008. Intangible Capital, 12(5), 1192-1220.

Veloso, C., Cuadra, A., Gil, F., Quiroz, Á., \& Meza, S. (2015). Capacitación en trabajadores: impacto de programa, basado en psicología positiva y habilidades sociales, en satisafacción, satisafacción laboral y clima. Interciencia, 737-741.

Volpi, B. (2014). El stres en las organizaciones del trabajo desde una mirada integrativa. (U. d. republica, Ed.) Repositotio del Instituto de Psicologia Social.

Weisbord, M. (1976). Estudios grupales y de organizaciónDiagnóstico organizacional: Seis lugares para buscar problemas con o sin una teoría. Estudios grupales y de organización, 430-447.

Weiss, D., Dawis, R., England, G., \& Lofquist, L. (1967). Manual for the Minnesota Satisfaction Questionnaire. Obtenido de Universidad de Minnesota: http://vpr.psych.umn.edu/ 
Zenteno, Á., \& Durán, C. (2016). Factores y practicas de alto desempeño que influyen en el clima laboral: analisis de un caso. 120-135. Obtenido de https://search.proquest.com/docview/1562070794 ?accountid=130858

Zou, M. (2015). Gender, work orientations and job satisfaction. Work, Employment and Society, 29(1), 3-22. 